Document downloaded from:

http://hdl.handle.net/10251/169907

This paper must be cited as:

Verdú, S.; Ruiz Rico, M.; Pérez Jiménez, AJ.; Barat Baviera, JM.; Grau Meló, R. (2020). Application of laser backscattering imaging for the physico-chemical characterisation of antimicrobial silica particles functionalised with plant essential oils. Journal of Food Engineering. 280:1-10.

https://doi.org/10.1016/j.jfoodeng.2020.109990

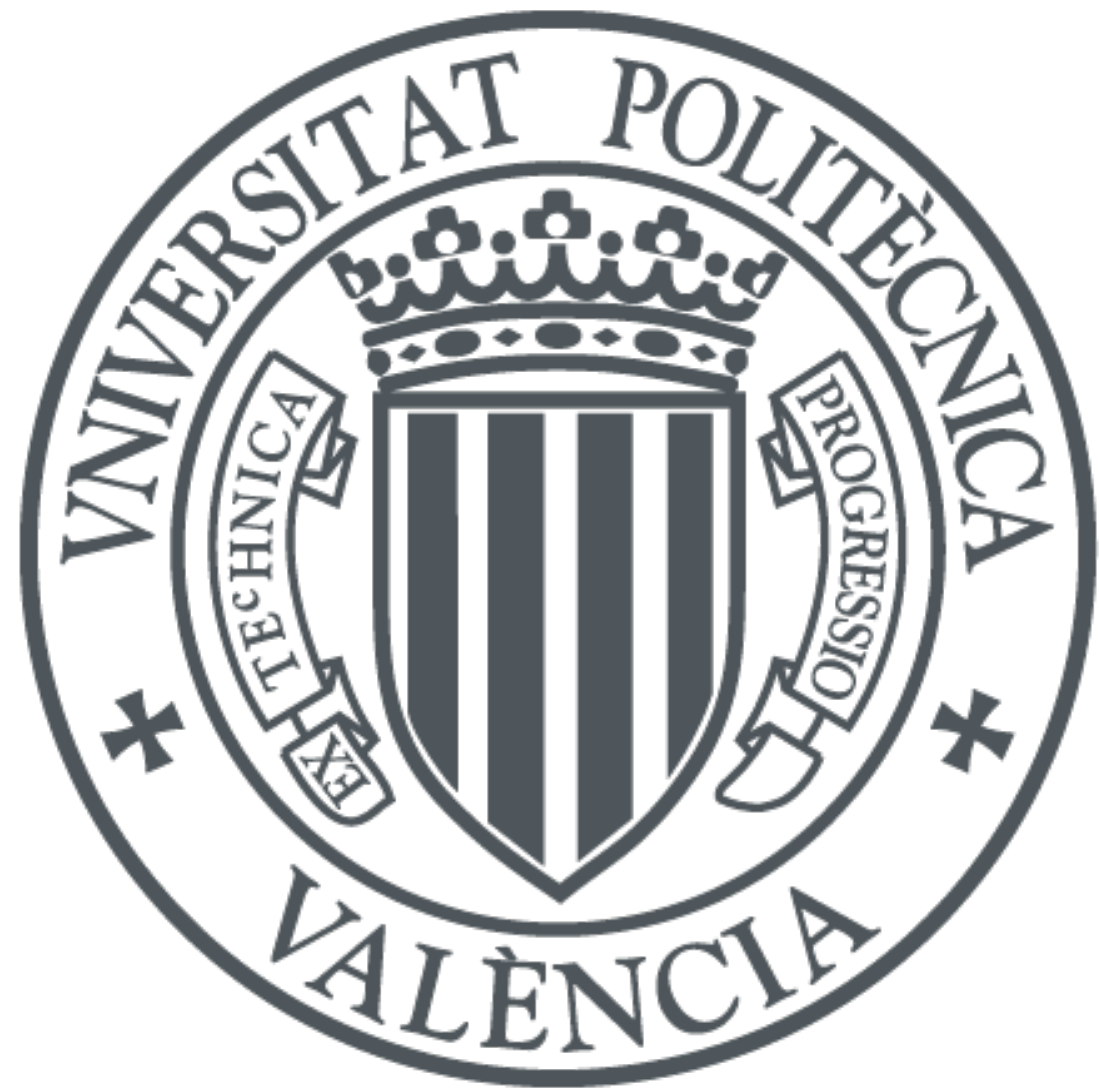

The final publication is available at

https://doi.org/10.1016/j.jfoodeng.2020.109990

Copyright Elsevier

Additional Information 


\title{
Application of laser backscattering imaging for the physico-chemical characterisation of antimicrobial silica particles functionalised with plant essential oils
}

\author{
${ }^{1}$ Samuel Verdú, ${ }^{1}$ Maria Ruiz-Rico, ${ }^{2}$ Alberto J. Pérez, ${ }^{1}$ José M. Barat, ${ }^{1}$ Raúl Grau \\ ${ }^{1}$ Departamento de Tecnología de Alimentos. Universidad Politècnica de València, Spain \\ ${ }^{2}$ Departamento de Informática de Sistemas y Computadores, Universidad Politècnica de València, Spain
}

\begin{abstract}
The capacity of orthogonal imaging applied to laser-backscattering for characterising antimicrobial particles based on immobilised essential oils was tested. Different particles were synthesised using various particle and oils. Samples were characterised physico-chemically and by an imaging technique. The technique recorded the generated patterns because of the laser-particles interaction during the sedimentation process. The series of images were transformed into an orthogonal image. Data extraction varied depending on the fragmentation degree of image length. After the multivariate analysis, the physico-chemical results showed variability due to particle size. That variability diminished the effect of oils for large sizes. The imaging data collected these properties, which could be used to recognise both particle size and oil type. Thus the prediction of the properties was successful. The position in the physico-chemical space of variance was also predicted. Hence this technique could complement a low-cost method to evaluate the properties of functionalised particles with oils.
\end{abstract}

Keywords: orthogonal imaging, laser backscattering, silica particles, essential oils, physicochemical characterisation 


\section{Introduction}

Emerging problems that negatively impact some synthetic antimicrobials and consumer health have been evidenced. Facts like abuse of antimicrobial substances due to inadequate traditional food-conservation methods increase the antibiotic resistant strains of bacteria and fungi, which all render the development of new strategies to prevent food spoilage and contamination necessary (Pisoschi et al., 2018). One of the main approaches in this area is to use naturally-occurring antimicrobial chemicals. Research into modifications in natural compounds has focused on changes in their properties. Some examples are solubility, dispersion across food matrices, avoiding volatility, etc. The main aim is to amplify the antimicrobial effect and to reduce the impact on products' organoleptic properties (Weiss et al., 2009). Accordingly, immobilisation of compounds on solid matrices has a high potential as regards the above-mentioned aim. This process provides incremented antimicrobial capacity from a small amount of compound compared to its free version. One of the main groups of these compounds is plant essential oils. Our research group has developed and tested these compound types by immobilising them onto particles of different materials, sizes and morphologies (silica) with successful results for both solid and liquid foods (Ribes, Ruiz-Rico, Pérez-Esteve, Fuentes, \& Barat, 2019; Ribes et al., 2017; Ruiz-Rico et al., 2017). The result is many possible particle types whose physico-chemical properties (zeta-potential, particle size distribution, surface area, uniformity, etc.) may vary significantly depending on the selected combinations. We observed that these properties could be determining factors for the effectiveness of a pre-designed antimicrobial. This effectiveness is associated with behaviour in relation to the food matrix. Therefore, controlling the properties of these compounds is a crucial aspect for their industrial and biotechnological applications (Dickinson, 2012). However, different equips and devices, and a relatively long time, are needed to acquire a complete data pool (particle size properties, zeta potential, thermos-gravimetric analysis, etc.).

So numerous methods can characterise properties related to particle size and distribution due to repercussions on other properties. Some of the most frequently used methods are the liquid sedimentation method, microscopy and laser diffraction scattering. The liquid sedimentation 
method has been widely used as the standard method (ISO 13317-4:2014). It is based on a direct mass measurement to give the mass distribution of the equivalent spherical particle diameter. Microscopic methods are relatively simple, but need a long measuring time when particle size distribution is wide. The particles tracking analysis (PTA) has reported good results by image analysis procedures to determine not only size properties, but also the behaviour of particles during sedimentation (Śliwa, Jarzębski, \& Szutkowski, 2015). Laser-based measuring techniques can be classified into three groups according to their measuring principle: local filter technology, Fraunhofer diffraction, and laser-backscattering (Emmerich et al., 2019). Laser-backscattering is the most employed technique for inline applications. One example is dynamic light scattering (DLS) (Yin, 2012). It measures a stable flow of dispersed particles to acquire information with a light detector. It requires accurate calibration from a direct method (Bell, Minelli, Tompkins, Stevens, \& Shard, 2012). Laser-backscattering methods have also been applied to model and characterise food properties, and to process both solid and fluid food matrices. These approaches are based on a simple device, which also includes image analysis procedures. In this case, the laser's interactions with samples are captured as diffraction patterns in digital images. The image capturing regime depends on the type of required information. Static patterns from different samples can be captured in single images to study the static properties of a given matrix. When the characterisation of a sample depends on the evolution of properties over time, many imaging captures should be carried out in a dynamic regime.

The patterns from these digital images are processed and transformed into numerical data. This information can be used to predict simultaneously food features and process parameters for nondestructive physico-chemical monitoring. Some examples of static characterisations with which we work are the prediction of rheological properties from vegetable-based creams (Verdú, Pérez, Barat, \& Grau, 2018) and the physico-chemical properties of biscuits with different fibre contents (Verdú, Barat, \& Grau, 2019a). The dynamic study of patterns allowed us to monitor the texture of milk during fermentation for yogurt production (Verdú, Barat, \& Grau, 2019b). In the present work, orthogonal imaging was applied to simplify the collection of laser patterns information with 
time by a dynamic analysis approach. This image mode means capturing information from the image sequence given by a dynamic process in a single image. Hence this approach reduces the imaging transform need and can improve previous applications to capture dynamic information over time.

This work focused on studying the application of orthogonal imaging for the physico-chemical characterisation of antimicrobial silica particles functionalised with plant essential oils.

\section{Material and Methods}

\subsection{Experiment procedure}

Figure 1. Scheme of the experiment. F1: Factor 1, particle size ( $a, b$ and $c)$; F2: Factor 2, essential oil type.

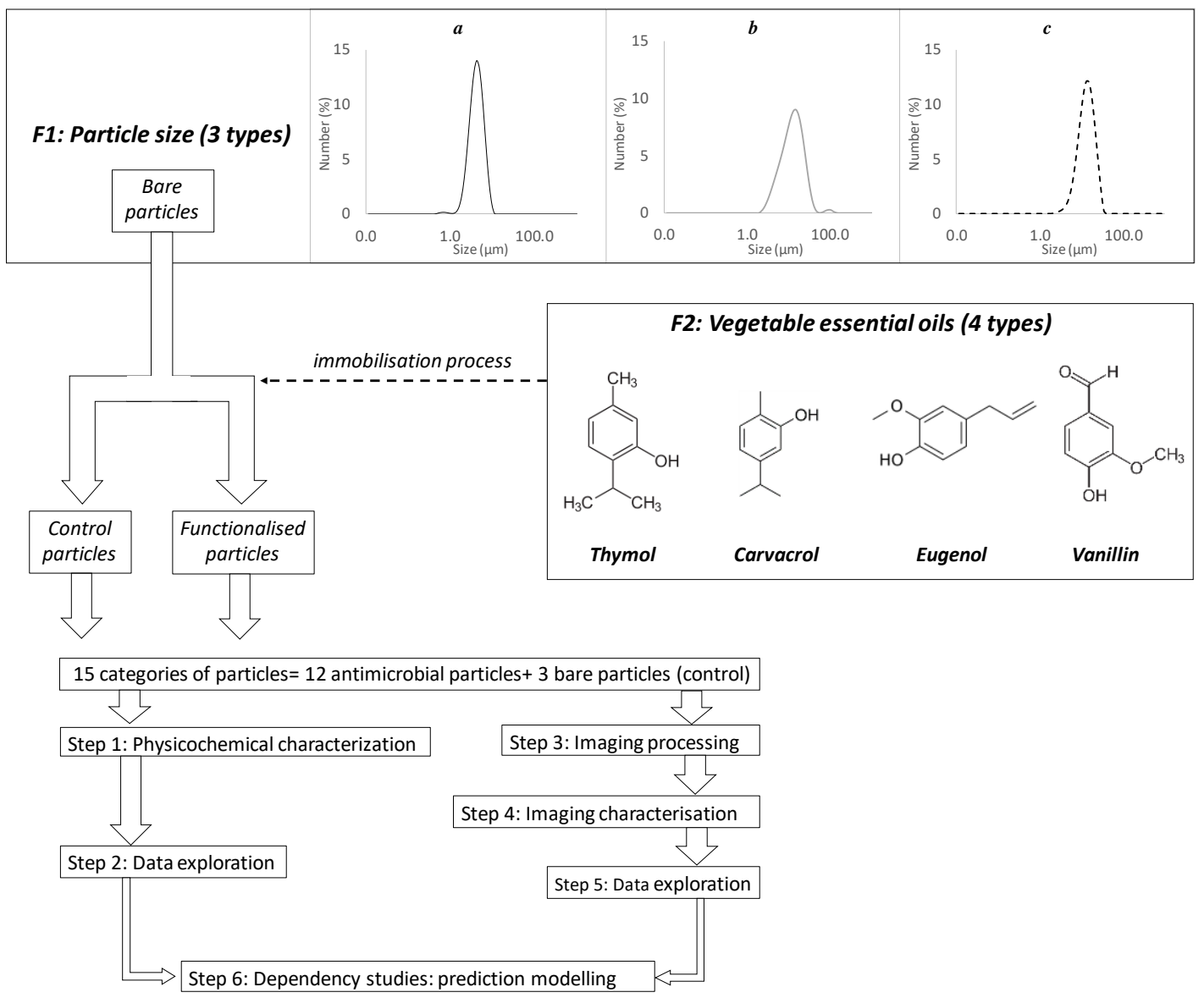

The experiment focused on characterising antimicrobial particles in terms of their physicochemical properties by orthogonal images applied to the laser-backscattering technique. In order 
to obtain wide variability in the properties of antimicrobial particles, they were manufactured by modifying some factors: particle size distribution, F1; type of immobilised essential oil, F2 (Fig. 1). F1 had three categories ( $a, b$ and $c$ ) of the used commercial silica particles, whose particle size distribution is represented in Fig. 1 and explained in Section 2.1.1. of Material and Methods. F2 had four different oils: eugenol, thymol, carvacrol and vanillin. Bare particles were included in the study as the control group. The combination of both factors provided 15 particle categories: 12 functionalised and three bare. The bare ones used as controls. Particles were characterised according to several physico-chemical analyses (thermo-gravimetric analysis, zeta potential, particle size distributions, etc.) (Fig. 1, Step 1). The physico-chemical variance generated by the combination of factors was explored by the multivariate statistical method Principal Component Analysis $(P C A)$. It provided a relation between the particle categories and the physico-chemical variables based on synthetic variables called principal components $(P C s)$, which capture the physico-chemical variance in reduced dimensionality (Fig. 1, Step 2). The coordinates within PCs were used as a characterisation map of the factors' influence. The next step was to study particles based on the images of the laser-particle interaction captured during the sedimentation process. Captured images were processed to acquire the most useful information (Fig. 1, Steps 3 and 4). Image information was also explored by $P C A$ to characterise particle categories (Fig. 1, Step 5). The last step was to study the relation between both data blocks by a Partial Least Square Regression (PLS-R) analysis to predict the physico-chemical properties of the particles from the image information (Fig. 1, Step 6).

\subsection{Particle functionalising}

\subsubsection{Reagents}

Different reagents were used to prepare the functionalised supports. Carvacrol ( $\geq 98 \% \mathrm{w} / \mathrm{w})$, eugenol $(99 \% \mathrm{w} / \mathrm{w})$ and thymol $(\geq 98.5 \% \mathrm{w} / \mathrm{w})$ were obtained from Sigma-Aldrich (Madrid, Spain) and vanillin (> 99\% w/w) was supplied by Ventós (Barcelona, Spain). They were used as bioactive compounds to prepare the supports functionalised with antimicrobial compounds. 
Acetonitrile, diethyl ether, dichloromethane and methanol were purchased from Scharlab (Barcelona, Spain), while chloroform and 2-butanone came from Sigma-Aldrich (Madrid, Spain), which were used as solvents for the derivatisation and grafting reactions. (3Aminoproppyl)triethoxysilane (APTES), paraformaldehyde, trimethylamine, sodium borohydride and $\mathrm{KOH}$ were purchased from Sigma-Aldrich (Madrid, Spain), and $\mathrm{NaCl}, \mathrm{MgSO}_{4}$, $\mathrm{H}_{2} \mathrm{SO}_{4}$ and $\mathrm{HCl}$ were obtained from Scharlab (Barcelona, Spain), which were the other reagents needed for the derivatisation of bioactive compounds.

Silica particles $a$ had an average $4 \mu \mathrm{m}$ particle size according to the product specifications of SYLYSIA® SY350/FCP, which came from Silysiamont (Milano, Italy). Silica particles $b$ had a average $25 \mu \mathrm{m}$ particle size according to the product specifications from Sigma-Aldrich (Madrid, Spain). The technical information on the specification sheet of particles $b$ indicated a particle size that fell within the 5-25 $\mu \mathrm{m}$ range. Silica particles $c$ had an average $10 \mu \mathrm{m}$ particle size according to the product specifications from Sigma-Aldrich (Madrid, Spain). These particles' size was between 5-15 $\mu \mathrm{m}$ in accordance with the specification sheet.

\subsubsection{Synthesis of the functionalised particles}

The functionalised silica microparticles lots were synthesised following a four-step synthetic procedure (García-Ríos, Ruiz-Rico, Guillamón, Pérez-Esteve, \& Barat, 2018). Firstly, the aldehyde derivatives of carvacrol, eugenol and thymol were prepared to add a second reactive moiety to molecules in order to keep the hydroxyl group free, which is essential for the antimicrobial activity of bioactive compounds (Gill \& Holley, 2006). Secondly, the unmodified vanillin and previously synthesised aldehyde derivatives were reacted with APTES to obtain alkoxysilane derivatives capable of being attached to the surface of silica microparticles in a third step. Finally, the imine bond of the alkoxysilane derivatives was transformed into an amine bond to stabilise the immobilised compounds. 
The carvacrol and thymol aldehydes were synthesised by direct formylation using paraformaldehyde, while eugenol aldehyde was synthesised following a Reimer-Tiemann reaction (Chen, Shi, Neoh, \& Kang, 2009).

The alkoxysilane derivatives were synthesised by a reaction run with APTES. In a typical synthesis run, the carvacrol, eugenol and thymol aldehyde derivatives and pure vanillin were suspended in dichloromethane, and APTES was added to the mixture at a molar ratio of 1:1 (essential oil: APTES). The mixture was stirred in reflux for $1 \mathrm{~h}$, and evaporated at reduced pressure to obtain the corresponding alkoxysilane derivatives.

To attach the alkoxysilane derivatives to the surface of the different mean sized silica microparticles $(5,10$ or $25 \mu \mathrm{m}), 10 \mathrm{~g}$ of the bare particles were placed in a round-bottomed flask in an inert atmosphere and suspended in $150 \mathrm{~mL}$ of acetonitrile. Then excess alkoxysilane derivatives were added and the mixture was stirred for $5.5 \mathrm{~h}$ at room temperature. After immobilisation, the stabilisation of the chemical bond of the alkoxysilane derivatives was carried out by suspending solids in $150 \mathrm{~mL}$ of methanol and running a reaction with excess sodium borohydride for $12 \mathrm{~h}$. The final solids were centrifuged, washed with distilled water $(\mathrm{pH} 4)$ and ethanol, and dried at room temperature in a vacuum for $12 \mathrm{~h}$.

\subsection{Physico-chemical characterisation of particles}

The bare and functionalised particles were characterised by different instrumental techniques. Size distribution and percentiles $d(0.1), d(0.5), d(0.9)$, volume-weighted mean $D[4,3]$, surfaceweighted mean $D[3,2]$, uniformity $U$ and specific surface area $S A$ were determined in a Malvern Mastersizer 2000 device (Malvern Instruments, UK). The Mie theory was applied by considering a refractive index of 1.45 and an absortion index of 0.01 . To determine the zeta-potential $(Z p)$, a Zetasizer Nano ZS (Malvern Instruments, UK) was used. Samples were dispersed in water at a concentration of $1 \mathrm{mg} / \mathrm{mL}$. Before taking each measurement, samples were sonicated for $2 \mathrm{~min}$ to preclude aggregation. The zeta-potential was calculated from the particle mobility values by applying the Smoluchowski model at $\mathrm{pH} 7$ and $20^{\circ} \mathrm{C}$. The degree of functionalisation of the 
particles was determined by a thermo-gravimetric analysis (TGA). The $T G A$ were carried out on a TGA/SDTA 851e Mettler Toledo balance (Mettler Toledo Inc., Schwarzenbach, Switzerland) in an oxidant atmosphere (air $80 \mathrm{~mL} / \mathrm{min}$ ) with a heating programme consisting of heating steps at $5^{\circ} \mathrm{C}$ per min from $25-1,000^{\circ} \mathrm{C}$. $T G A$ was expressed as the percentage of organic matter $(\%$ o.m). All the analyses were done in triplicate. The above-mentioned variables formed part of the physico-chemical data block.

\subsection{Imaging device}

Orthogonality is the notion of perpendicularity in linear algebra. In this case, dynamic light intensity information (with time) was required to generate an orthogonal image of each sample. Hence the obtained orthogonal images represented a pixel position on the $\mathrm{X}$-axis and time on the Y-axis; i.e., include in an image the variation of light intensity for each pixel with time as perpendicular axes (Fig. 2). To obtain that continuous information from the diffraction patterns generated by the laser-particles interaction during the sedimentation process, a low-cost device consisting in a red laser diode and a digital camera connected to a computer was built. Fig. 2A shows the device setup.

The capture system of the device for image analysis was a digital Logitech C920 camera with a CMOS sensor that operates at a resolution of 230401535. The angle of view (AOV) of the lens was $78^{\circ}$, which gave a field of view (FOV) of approximately $100 \mathrm{~mm}$ at the work distance. Camera exposure and gain settings were configured to the auto mode. The camera included an HD video working in the H.264 high quality format so that the 198001080 obtained images would present minimum lost. The device was placed inside a dark cabin to keep it away from light, and was vertically placed $15 \mathrm{~cm}$ over the sample surface. The laser pointer $(650 \mathrm{~nm}, 50 \mathrm{~mW}, 3 \mathrm{~mm} \bullet)$ was perpendicularly placed $20 \mathrm{~cm}$ under samples by emitting to the central zone of the bottom surface. Both the laser diode and camera (HD cam Logitech C920) were low-cost components, and were connected to a conventional computer that ran Linux. The capture temperature was $20^{\circ} \mathrm{C}$. Samples were prepared as particle suspension $(0.7 \mathrm{~g}$ particles, $2.3 \mathrm{~mL}$ distilled water $)$ on a transparent well 
plate (12-well plate, $21.2 \mathrm{~mm} \varnothing, 6.9 \mathrm{~mL}$, Corning® Costar®) and shaken for 1 minute at $250 \mathrm{rpm}$ (Orbital shaker Ivymen® system). When the stirrer was stopped, the laser beam was pointed through a flat transparent well plate to interact with the suspension during the sedimentation process. The system was controlled by a specific program that automatically triggers the camera by software to take images at a rate of 1 image/sec. for $15 \mathrm{~min}$ (Fig. 2A). Nine hundred images from each sample were obtained at the end of the sedimentation process. Twenty samples of each particle category were analysed.

\subsection{Imaging processing}

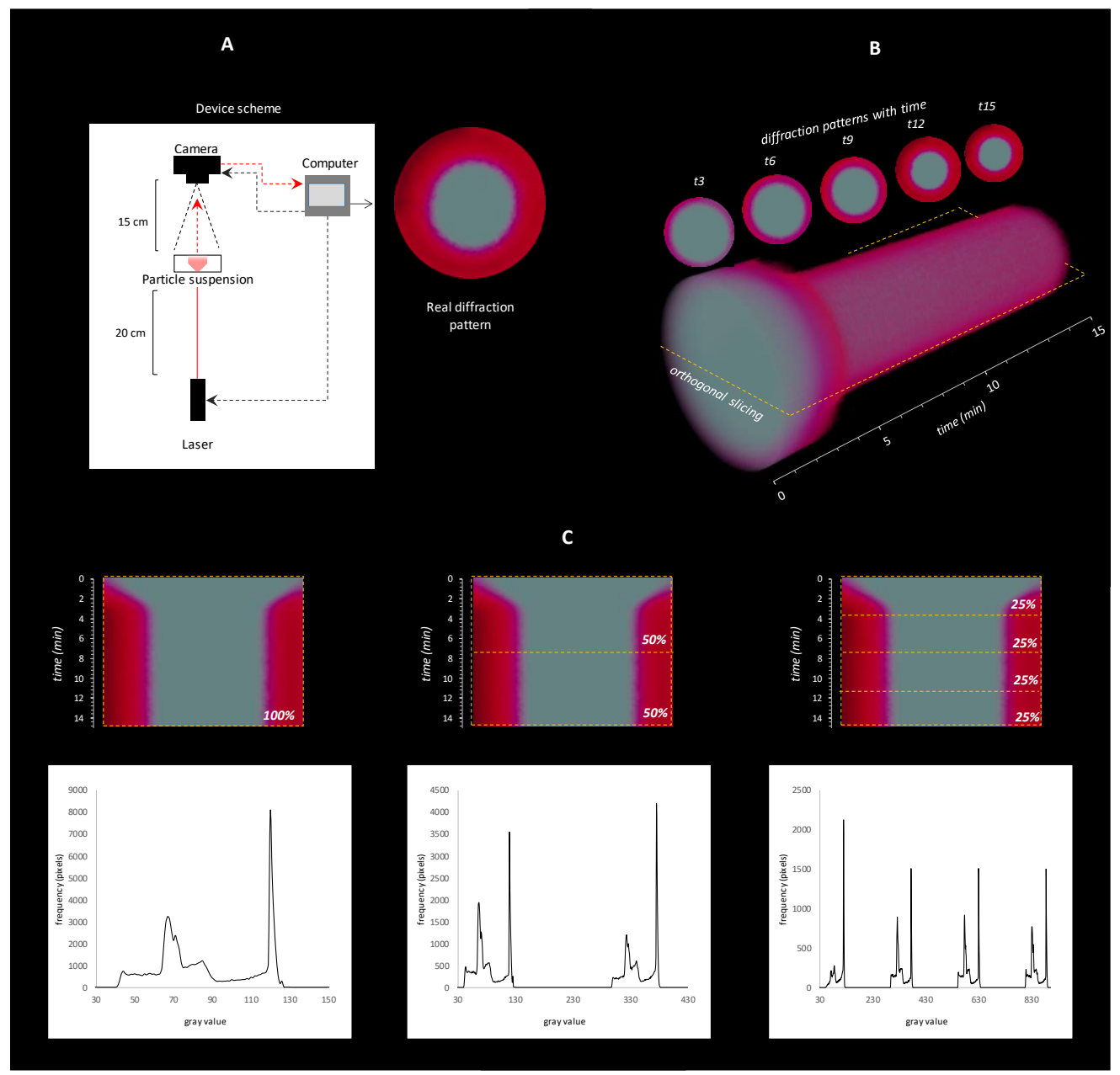

Figure 2. Image processing and data extraction. A: image device scheme; B: evolution of the diffraction pattern with time and orthogonal image extraction; $\mathrm{C}$ : the orthogonal image fragmented into different degrees and the data obtained from fragments (histograms). 
An own software was developed to quickly and accurately process images. The software was developed in C language using the OpenCV 2.4.1 image processing libraries (Bradski, 2000). It runs on a computer with Linux Ubuntu 16.04 as the operating system. The procedure was run as follows:

1. Creation of a stack from the entire process: the 900 images captured during the process were joined to an image stack, where the Z-axis represented time. This resulted in a three-dimensional column where the variation of diffraction patterns was collected with time (Fig. 2B)

2. Orthogonal images extraction: variations in light intensity throughout the process were captured in orthogonal images to simplify the monitoring process from 900 images to 1 image. That image was a slice across the time axis of the stack that was $21.2 \mathrm{~mm}$ wide (diameter of the well) on the X-axis, which crossed the central point of the diffraction pattern (Fig. 2C). The obtained images represent the pixel position on the $\mathrm{X}$-axis and time on the $\mathrm{Y}$-axis

3. Data extraction from the orthogonal images: data were extracted based on the greyscale histograms at different degrees of image fragmentation. Images were transformed into a greyscale ( 8 bits) to simplify the collected information. Afterwards, they were divided into different fragments based on the \% image lengths (time, Y-axis), which were 100\%, $50 \%, 25 \%, 10 \%$ and $5 \%$ of the time axis. Thus images were fragmented in different areas prior to data extraction. Histograms were extracted, which collected information on frequency (expressed as pixels) for each grey value (256 values) within a given fragment, defined by the degree of fragmentation. Therefore, the amount of data depended on the degree of fragmentation. For example, the $100 \%$ image length had one histogram from the entire image, $50 \%$ went to two histograms from each half, $25 \%$ to four histograms, etc. (Fig. 2C). Histograms were organised in a data matrix as spectra formed by 256 variables for the multivariate statistical analysis. When the degrees of fragmentation had more than one histogram, they were serially organised. 
This algorithm shows in detail the applied process:

INPUT: $\{\mathrm{Ii}[\mathrm{C}][\mathrm{R}] \mid \mathrm{i}=1 . . \mathrm{N}\}$ set of $\mathrm{N}$ captured images of size $\mathrm{C} \diamond \mathrm{R}$

- Create stack matrix $M[N][C][R]$ initialized as:

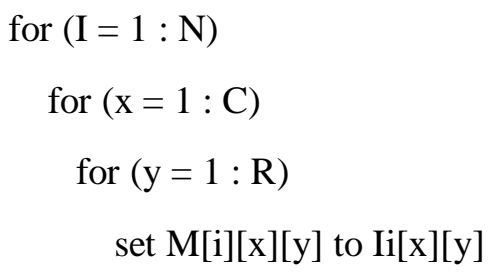

- Extract orthogonal image $\mathrm{O}[\mathrm{C}][\mathrm{N}]$ as:

for $(\mathrm{i}=1: \mathrm{N})$

set $(\mathrm{x} 0, \mathrm{y} 0)$ to the central point of the diffraction pattern in image Ii

for $(\mathrm{x}=1: \mathrm{C})$

set $\mathrm{O}[\mathrm{x}][\mathrm{i}]$ to $\mathrm{M}[\mathrm{i}][\mathrm{x}][\mathrm{yo}]$

- Compute fragmentation sets of image $\mathrm{O}$ with $\mathrm{f}=\{1,0.50 .25,0.1,0.05\}$ as:

set $\mathrm{O} 1=\{\mathrm{O}\}$, and successively,

set $\mathrm{Of}=\{\mathrm{F} 1, \ldots, \mathrm{F} 1 / \mathrm{f}\} \mid \mathrm{Fi}$ are of size $\mathrm{C} \diamond \mathrm{N}^{\prime}$ with $\mathrm{N}^{\prime}=\mathrm{f} . \mathrm{N}$, and $\mathrm{Fi}=\mathrm{O}[1: \mathrm{C}]\left[1+(\mathrm{i}-1) . \mathrm{N}^{\prime}: \mathrm{i} . \mathrm{N}^{\prime}\right]$

- Compute gray histograms of each image in sets $\{\mathrm{Of}\}$

OUTPUT: $\{\mathrm{Hf}\}$ sets of histograms of sets images $\{\mathrm{Of}\}$

\subsection{Statistical analysis}

The physico-chemical and image information were explored and compared after applying multivariable statistical procedures to reduce dataset dimensionality. To this end, the multivariate unsupervised statistical method $P C A$ was used to compare the variance collected by not only the physico-chemical variables, but also the image information from the antimicrobial particles. A $P L S-R$ was used to evaluate the dependence between the image information and the physicochemical properties of the tested antimicrobials. This method was used to carry out the linear regressions models between both datasets, which were evaluated based on the $R^{2}$ of prediction. The prediction models of the physico-chemical properties were run by using the image data from all the samples and degrees of image fragmentation. Samples were divided into a training batch (60\% of samples) and a testing batch ( $40 \%$ of samples). These procedures were run with the PLS Toolbox, 6.3 (Eigenvector Research Inc., Wenatchee, Washington, USA), a toolbox extension in the MATLAB 7.6 computational environment (The Mathworks, Natick, Massachusetts, USA). 


\section{Results and Discussion}

\subsection{Physico-chemical characterisation}

A PCA was carried out to simultaneously study the physico-chemical properties of particles from the analysed variables (Fig. 3A). This analysis provided a geometrical space of variance from which the relations between both physico-chemical properties and categories could be studied in an extensive lot of particles. This space of variance could represent a space formed by a given lot of particles used in industry or a laboratory from which its properties could be interpreted after model training.

The results showed the clustering of different particle sizes (F1) across $P C 1$ (X-axis). Sizes were placed from a negative zone of the axis to a positive one in this order: $a, c$ and $b$ (Fig. 3A, circumferences). This principal component had $48.9 \%$ of total variance, which meant that the differences in properties due to particle sizes generated almost half the total variance. The placement of the formed clusters also presented decreasing dispersion, with the maximum for $a$ and the minimum for $b$. The variance generated by particle size could be explained by $S A$ and $D[3,2], d(0.1)$ and $d(0.5)$. These parameters had a high explanation load for $P C l$. When particle size increased, $S A$ decreased. This revealed the importance and inverse evolution of $S A$ and the size distribution descriptors in spite of the immobilisation process.

Moreover, $P C 2$ had $27.8 \%$ of total variance. This principal component was related to the variance produced by the essential oil types, principally for particle size $a$. For the other sizes, no pattern for essential oils placement was observed. $P C 2$ had a very high explanation load by $U, d(0.9)$ and $D[4,3]$. These results indicated that the essential oil type (F2) had a stronger influence on properties when particle size was smaller. This meant that the immobilisation process of $T h$ and E provided higher $U, d(0.9)$ and $D[4,3]$ to particles when size was $a$.

Moreover, particles $b$ grouped in a small space and showed less dependence on essential oil type. This did not mean that there were no differences in their properties. However in that space of variance, size $b$ had a reduced proportion of it. When a $P C A$ with only size $b$ was run (Fig. 3B), 
the differences in the properties between the used essential oils were more easily observed. Size effect had no influence in that case, where $T h$ and $C r$ were the only categories to present a similar placement in this variance space. This space of variance represented the map of the physicochemical behaviour of the synthesised particles according to F1 and F2. This map can be modified given the interest in such properties by means of other modifications.
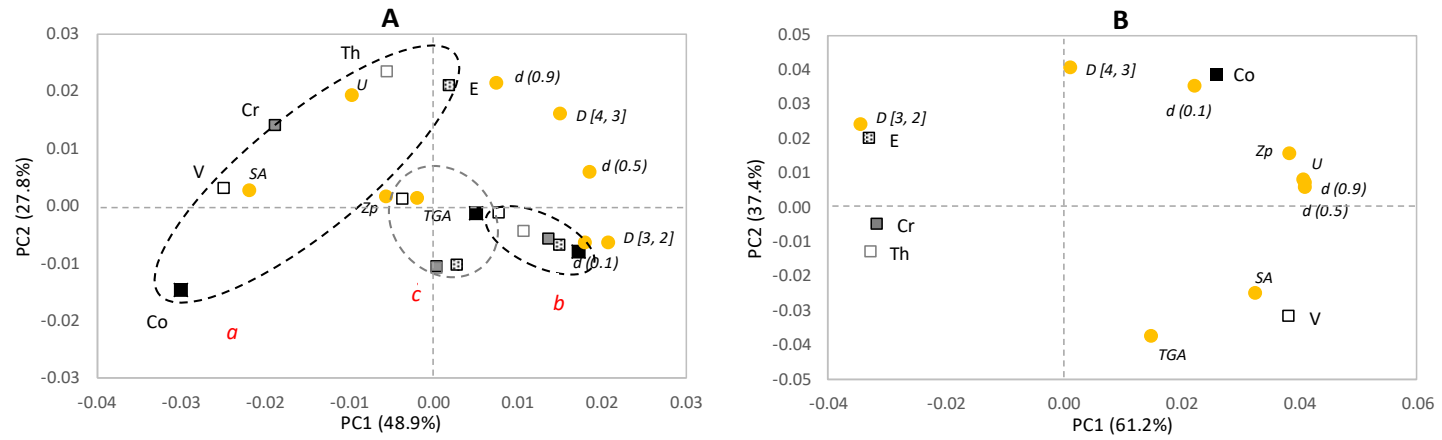

Figure 3. Physico-chemical characterisation of particles. Variance space generated from the multivariate analysis of the physico-chemical variables $(P C A)$. A: variance space for all the particle categories; $\mathrm{B}$ : variance space for the particles of size $b$. Yellow points denote the physico-chemical variables. Circumferences depict clusters of sizes. Percentiles: $d(0.1), d(0.5), d(0.9)$; volume-weighted mean: $D[4,3])$; surface-weighted mean: $D[3,2]$; uniformity : $U$; specific surface area :SA; zeta-potential: $Z p$, degree of functionalisation: $T G A ; C o$ : control (bare particles); $C r$ : carvacrol; $E$ : eugenol; $T h$ : thymol; $V$ : vanillin.

\subsection{Imaging processing and data exploration}

The orthogonal images obtained from the laser-particles interaction during the sedimentation processes were extracted to visualise their properties. Afterwards, data were extracted for the exploration process. Fig. 4 contains the images obtained from the process for each particle size (F1) and essential oil (F2) combination. The generated patterns presented different morphologies across the temporal axis, which were affected by both F1 and F2. There were two zones: saturated and diffused. The first is represented by the intense zone in the middle of the patterns, and the second by the fuzzy red contour. 
Common behaviour was the maximum light width at the beginning of the process. That width changed with time in different ways according to particle type until a constant shape appeared, from which point the sedimentation process ended. This behaviour showed how laser diffraction was initially large due to the high concentration of dispersed particles across the continuous phase. Dispersed particles interacted with radiation to produce marked changes in direction, followed by more diffuse light at the initial times. Thus diffraction reduced with the sedimentation process, and showed the observed curves due to the lower concentration of the dispersed particles because of increased sediment material. It was noteworthy that there were cases in which diffraction and absorption of light were so high that only the diffused zone was observed without the saturated one (Fig. 4, V).

Bare particles $(\mathrm{Co})$ displayed the particle size change effect. Co-A presented a column of the saturated zone with a marked width throughout the process. However, whereas when particle size increased $(C o-B)$, sedimentation became faster, and an asymptotic beginning was observed between 4-6 minutes. Moreover, the incorporation of essential oils brought about major changes in the patterns at all particle sizes. Accordingly, vanillin $(V)$ seemed to have a strong effect on behaviour, while thymol $(\mathrm{Th})$ and carvacrol $(\mathrm{Cr})$ were more similar to $\mathrm{Co}$, principally at particle sizes $b$ and $c$. 


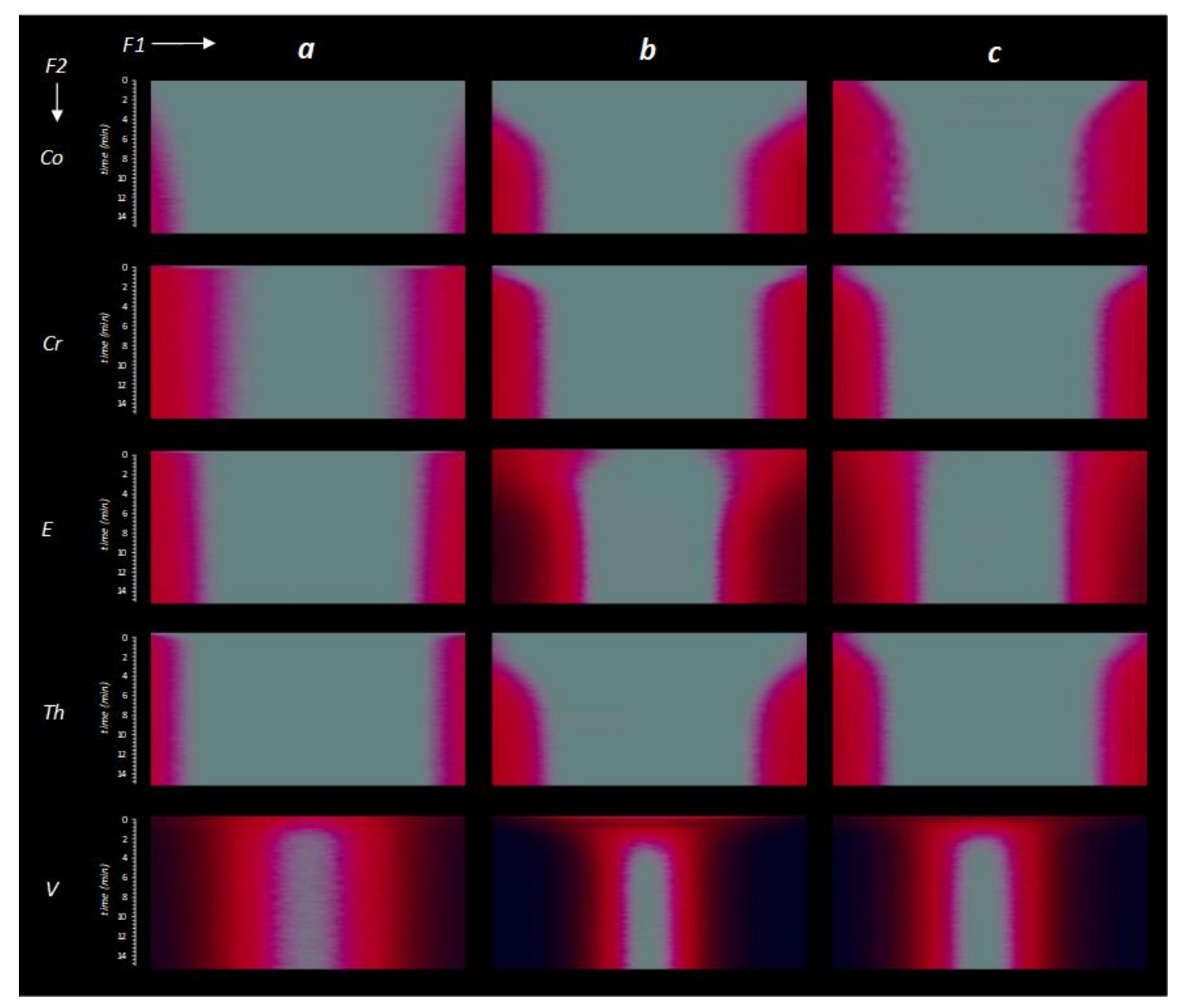

Figure 4. Orthogonal images of the laser-particles interaction during the sedimentation process. F1: particle sizes $a, b$ and $c$; F2: essential oil type $C o$ : control (bare particles); $C r$ : carvacrol; $E$ : eugenol; Th: thymol; $V$ : vanillin.

To facilitate the study of the observed effects, the orthogonal images were processed to extract multivariable information in the form of histograms at different image-fragmentation degrees, as explained above. The effect of factors on the extracted data was explored following the differences observed in the space of variance obtained in the $P C A$ from the physico-chemical analysis (Fig. 3). Previously, an increasing particle size seemed to narrow the differences between the physico-chemical properties of the functionalised particles, and $b$ size was the most evident one. Therefore, imaging information seemed to collect enough variance to differentiate particle categories in the same way as the studied physico-chemical variance spaces. These differentiations were dependent on the degree of image fragmentation. Fig. 5 shows some $P C A$ spaces obtained from the imaging data analysis from the group with the smallest observed 
differences: size $b$. Fig. 5A and 5B show the variance spaces to study information within the size $b$ particles using the minimum and maximum fragmentation levels (100\% and 5\% image length, respectively). For the minimum fragmentation (Fig. 5A), essential oils could be differentiated, principally $V$. $E$ was also placed away from the other oils, but came close to the control particles $(\mathrm{Co})$. $\mathrm{Th}, \mathrm{Cr}$ and $\mathrm{Co}$ were placed around the same position. In this case positions were in accordance with those observed in the patterns from the orthogonal images in Fig. 3 and 4, where the most differentiated ones were $V$ and $E$, while $T h$ and $C r$ presented very similar patterns for size $b$. When the same study was done using the data obtained from the maximum degree of fragmentation (the 5\% image length), bigger differences among categories were observed (Fig. 5B). Once again $V$ and $E$ were the most differentiated ones, but a more marked differentiation was seen in this case among $C o$, $T h$ and $C r$.

After obtaining this result, the differentiation capacity between particle sizes was also studied, but using only $\mathrm{Co}$, $\mathrm{Th}$ and $\mathrm{Cr}$. Those categories were selected because they represented the most unfavourable cases in terms of differences in behaviour. The spaces of variance generated after the PCA studies are shown in Fig. 5C-5D. Studies were done following a previous study about the degree of fragmentation. Fig. 5C shows the $P C A$ space generated after analysing the data from the minimum degree of fragmentation (the 100\% image length). No differentiation was observed for most categories, only for $C o$ and $C r$ at particle sizes $c$ and $a$, and a large cloud of samples was placed around the same place within the coordinates. Conversely, and following the previous study, differentiation of categories was observed when the degree of maximum fragmentation was used (Fig. 5D). This result agrees with other studies about classifying different particles using diffraction patterns such as Wang et al., (2019), who also used an imaging analysis of the diffraction patterns obtained from the interaction between particles and coherent light. In that case, the difference lay in distinct light intensities, and a continuous flow of fluid with suspended particles was used to classify particles with different morphologies.

Therefore, the results showed how the studied sedimentation process of particles was affected by both F1 and F2, which caused differences in the laser interaction that were successfully collected 
by the patterns of orthogonal images. These differences could be used to characterise all the categories, although the information from images had to be processed to obtain the target in those cases with the smallest differences.
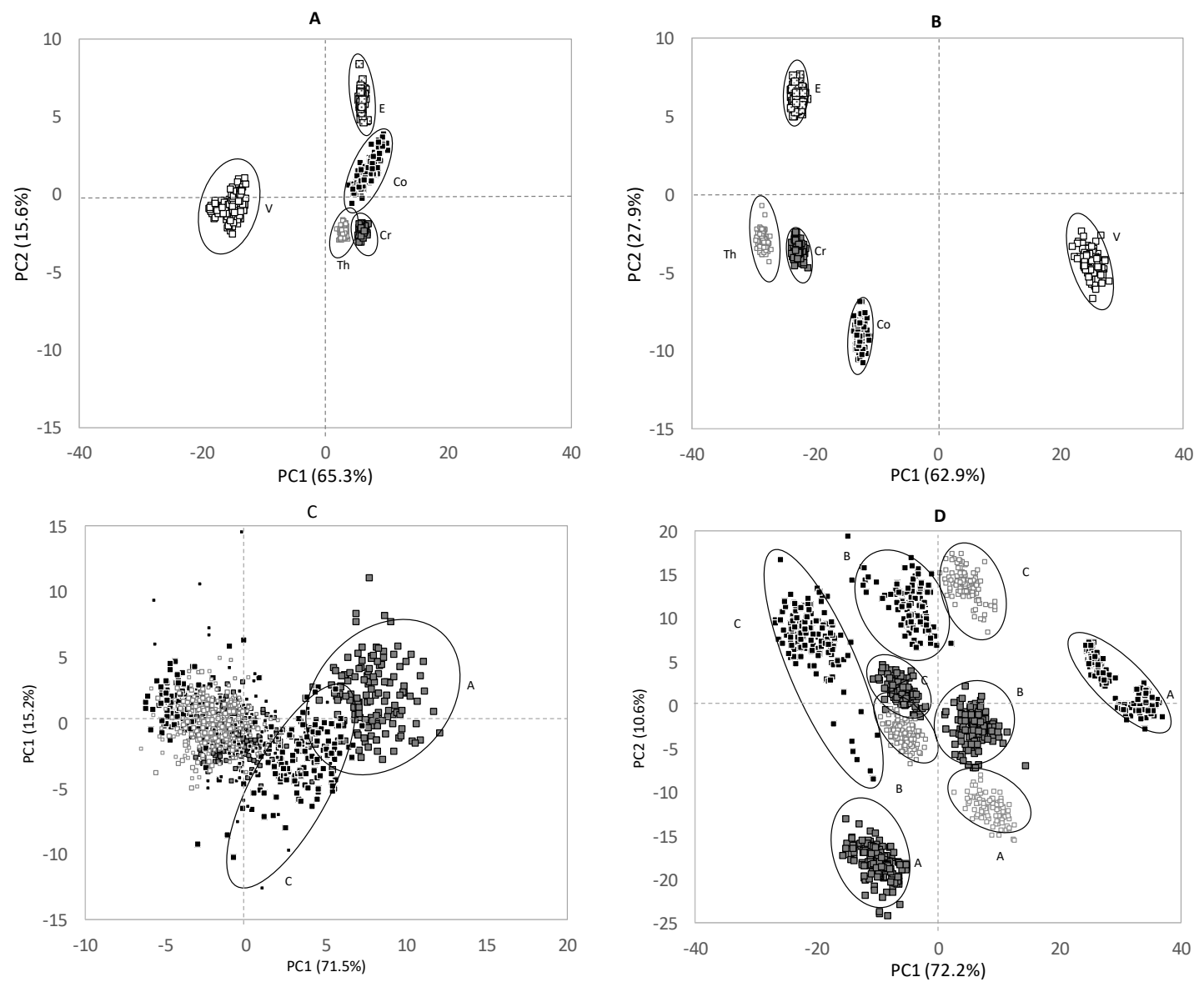

Figure 5. Image data exploration. A: $P C A$ of particle size $b$ using the minimum degree of fragmentation (the $100 \%$ image length); B: $P C A$ of particle size $b$ using the maximum degree of fragmentation (the $5 \%$ image length); $\mathrm{C}: P C A$ of all the particle sizes ( $a, b$ and $c$ ) for $C o$, Th and $C r$ using the minimum degree of fragmentation (the $100 \%$ image length); D: PCA of all the particle sizes ( $a, b$ and $c$ ) for $C o$, Th and $C r$ using the maximum degree of fragmentation (the 5\% image length); $C o$ : control (bare particles); $C r$ : carvacrol; E: eugenol; Th: thymol; $V:$ vanillin.

\subsection{Prediction studies}

After observing the capacity of imaging data to characterise particle categories, dependency studies were carried out to test the relation between that data block and the physico-chemical properties data block. The imaging data block from each degree of fragmentation was used to 
singly predict each physico-chemical variable and the scores (PC1 and $P C 2)$ obtained in the physico-chemical PCA analysis (Fig. 3). The prediction of the place in the physico-chemical variance space was done to test the estimation of coordinates to simultaneously know the physicochemical properties for the relations from each pre-established placing.

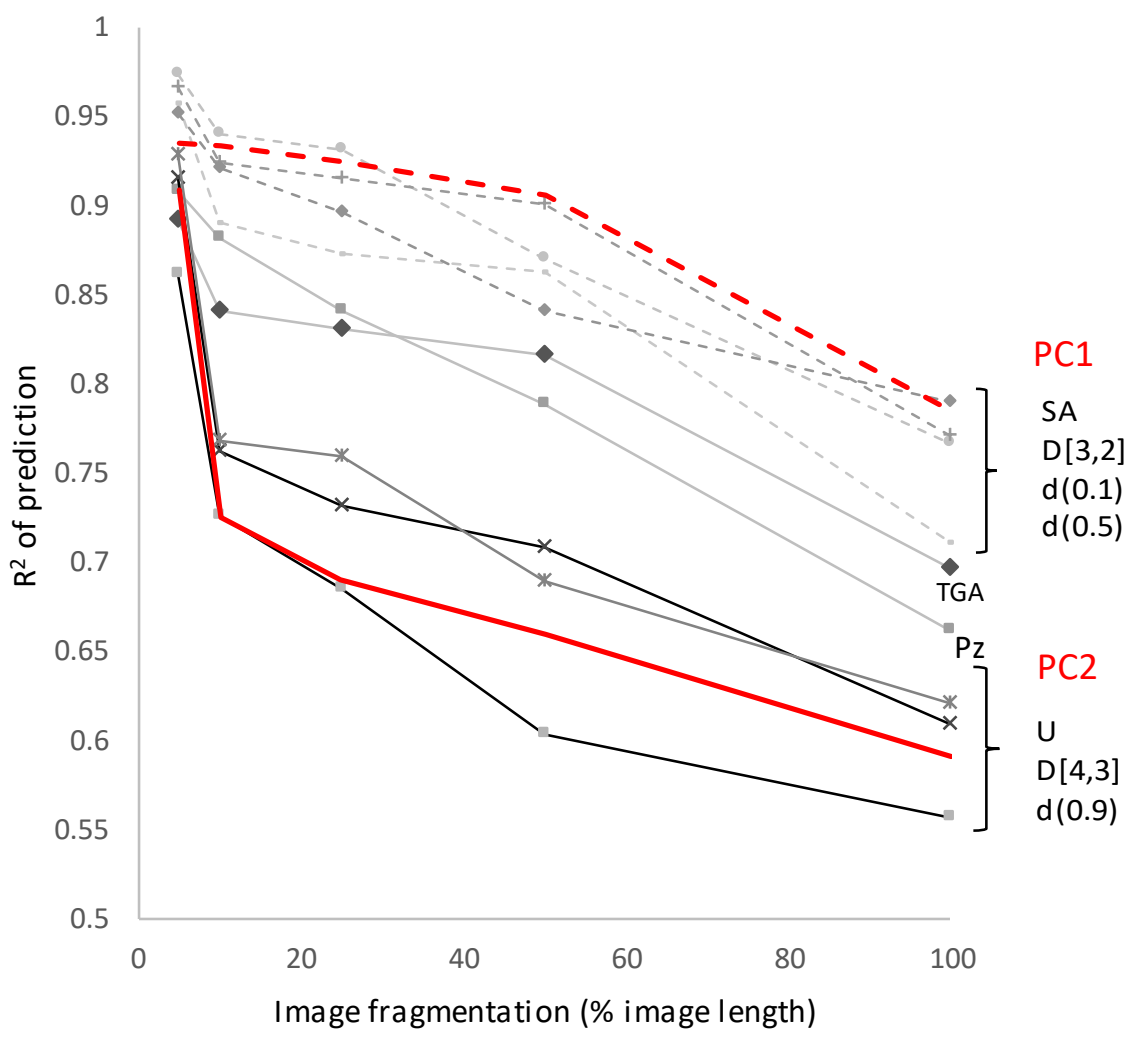

Figure 6. Dependency studies among the image data, single physico-chemical variables and principal components from the physico-chemical space of variance. The evolution of $R^{2}$ prediction with degree of image fragmentation (the $\%$ image length). Percentiles: $d(0.1), d(0.5), d(0.9)$; volume-weighted mean: $D[4,3])$; surface-weighted mean $: D[3,2]$; uniformity : $U$; specific surface area :SA; zeta-potential: $Z p$, degree of functionalisation: $T G A$. The different parentheses indicate the variables related with $P C 1$ (red dotted line) and $P C 2$ (red line) in the physico-chemical space of variance in Fig. 4A.

The $R^{2}$ prediction results from the $P L S$ studies are found in Fig. 6, which represents the evolution of the prediction coefficient across the degree of image fragmentation. Predictions showed an increasing coefficient with degree of fragmentation. The minimum degree of fragmentation (the 
$100 \%$ image length) had an $R^{2}$ of between $0.57-0.79$. The increase in coefficients seemed to denote a linear tendency until the degree of fragmentation of the $10 \%$ image length. A generalised increase in slope was observed, mainly for the variables with a low $R^{2}$ for the $100 \%$ image length. Thus the range of coefficients at the maximum degree of fragmentation (the 5\% image length) was 0.86-0.97. The variables that explained more of the variance captured for $P C 1$ and for themselves (Fig. 3A) were predicted with smaller errors from the $100 \%$ image length: $S A, D[3,2]$, $d(0.1)$ and $d(0.5)$. They appear at the top of the plot in Fig. 6, while $U, d(0.9)$ and $D[4,3]$, respectively related to $P C 2$, and $T G A$ and $Z p$, started from a low $R^{2}$ prediction and also obtained a lower prediction coefficient at the maximum \% image length (bottom of the plot). So the variables that were directly characterised by the effect of F1 (particle size) were better predicted. This result was probably due to their wider variance of $\mathrm{F} 1$ compared to the variables related with F2 (essential oil), although they all presented an $R^{2}$ prediction above 0.85 .

Fig. 7A shows the physico-chemical space of variance from Fig. 3A, which includes the coordinates predicted for $P C 1$ and $P C 2$ at the 5\% image length. Dispersion considerably increased for particle sizes $b$ and $c$ and, due to the similar placement, the model showed some confusion for precise predictions. The reduced variance among the bigger particle sizes, when the complete model was used, made its differentiation in the PCA space difficult. In order to improve the results, model complexity was reduced by studying a model for each factor, and their $P C 1$ and $P C 2$ prediction capacity was tested. As the differences between the properties of each particle size and essential oil could limit the accuracy of the studied common prediction model, different models were tested by isolating each particle type; that is, a specific prediction model was tested for each particle size and essential oil type. The prediction results for each particle size model are represented in Fig. 7B. The places in the variance physico-chemical space were successfully predicted for particles $a$ and C. Most $b$ particles were placed around the correct coordinates, but some of them overlapped others. Moreover, the single models for each essential oil provided the most accurate prediction approaches (Fig. 7C). The placing of the essential oil types for particles $a$ was correct, with little dispersion shown for them all. The dispersion in particles $b$ and $c$ also 
drastically reduced, where the different oils in these coordinates were differentiated. This result showed how precision increased with reduced model complexity because the results improved when the physico-chemical variance spaces from size $b$ (Fig. 4B) were predicted, despite a model with all the essential oils for that size being used (Fig. 7D). Fragmenting images provided more information from which the characterisation of particles could be improved. Following this result, it was possible to improve the coefficients with a more drastic degree of image fragmentation (e.g. $2 \%$ image length). However, the increase in data volume could mean disadvantages for data handling because if the 5\% image length had 20x256 variables, then the $2 \%$ image length would represent 50x256 variables.

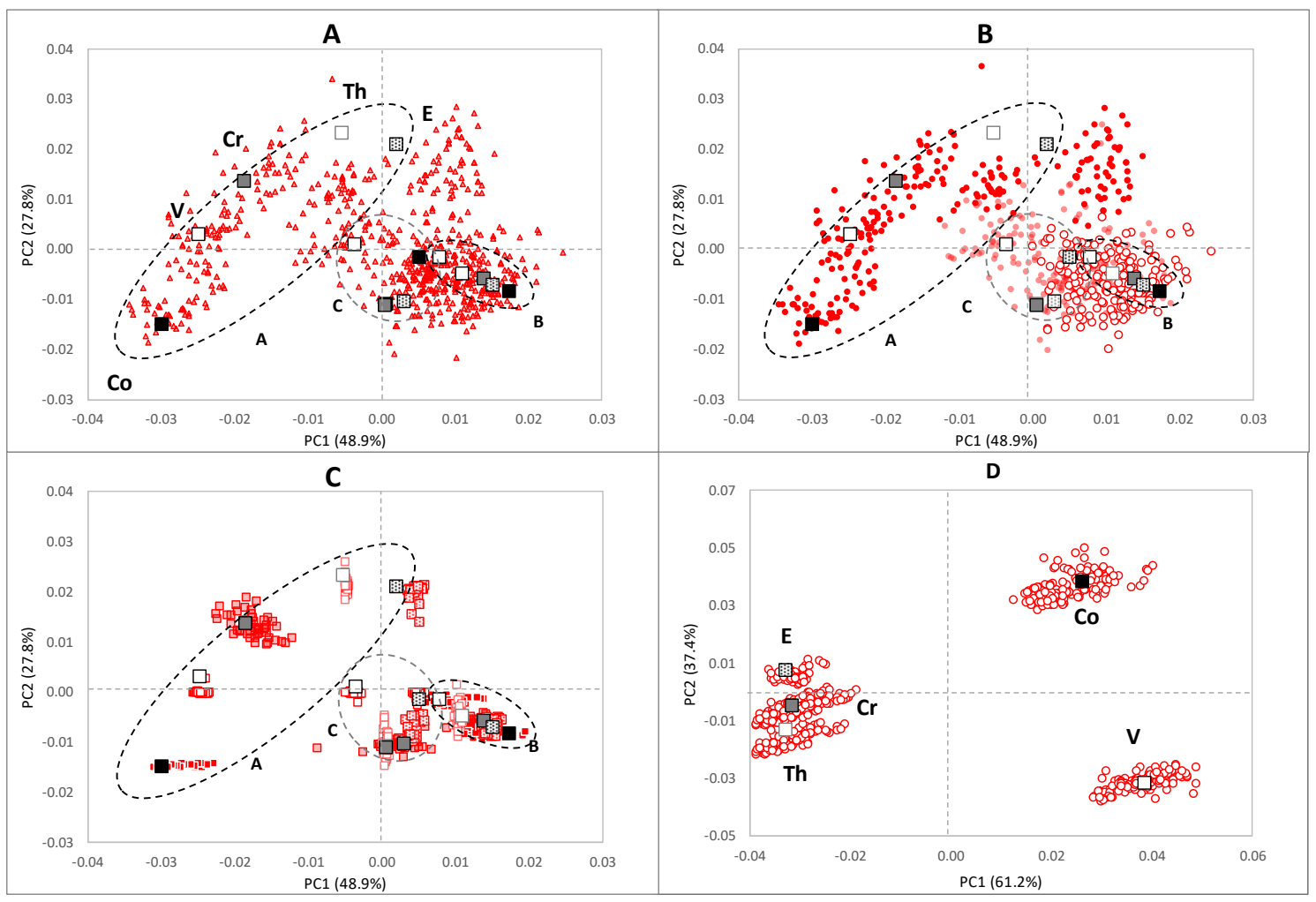

Figure 7. Prediction studies of the coordinates in the physico-chemical space of variance from the image data (the 5\% image length). A: prediction using a common model; B: prediction using a single model for each particle size; C: prediction using a single model for each essential oil; D: prediction for the physico-chemical space of variance for particle size $b$ (Fig. 3B). $\square$ : vanillin; $\square$ : thymol; $\square$ : carvacrol; $\square$ : eugenol; $\mathbf{\square}$ : control: coordinates observed in the physicochemical space of variance (Fig. 3A); $\Delta$ data predicted from the common model; $\bullet$ : data predicted with the particle $a$ size model; ○: data predicted with the particle $b$ size model; $\bullet$ : data predicted with the particle $c$ size model. 
Therefore, a relation between particle behaviour during sedimentation and the data obtained from the employed imaging technique was evidenced via the possibility of predicting physico-chemical properties. The monitored process provided information about both particle size and essential oil type, but increased the degree of fragmenting image, while reducing the complexity of models could provide more accurate results in a given space of physico-chemical variance.

\section{Conclusions}

Orthogonal imaging applied to the laser-backscattering technique could be used to characterise the physico-chemical properties of different categories of functionalised silica particles with plant essential oils. Differences in particle size generated most of the observed physico-chemical variance, and diminished the effect of the different essential oils when size increased. Imaging technique information could be used to characterise particles in size and essential oil type terms. A common model made by using information from all the particle categories reported successful prediction coefficients for single physico-chemical variables, mainly for those most affected by particle size. The prediction coefficients were in accordance with the degree of image fragmentation, which rose following a reduction in $\%$ image length. Moreover, the prediction of the coordinates in the common physico-chemical variance space generated by $P C A$ can be done using the common model. However, precision reduced with increasing particle size. Reducing the model's complexity by generating new models using only either particle size or essential oil improved the precision of sample coordinate predictions.

Therefore, this image technique could contribute to a low-cost application based on laser backscattering to simultaneously evaluate the physico-chemical properties of functionalised particles with essential oils to train the prediction models formed by different particle categories. Future studies will focus on testing other immobilised functional molecules which our group is testing in different food matrices, like organic acids, as well as particles made from different materials. New protocols will also be studied to extract information from orthogonal images to develop more precise models with greater complexity. 


\section{Acknowledgement}

The authors gratefully acknowledge the financial support from the University Polytechnic of Valencia for Programme "Ayudas para la Contratación de Doctores para el Acceso al Sistema Español de Ciencia, Tecnología e Innovación, en Estructuras de Investigación de la UPV (PAID-10-17)”, "Generalitat Valenciana" for their postdoctoral fellowship (APOSTD / 2019/118) and the Ministerio de Ciencia, Innovación y Universidades, the Agencia Estatal de Investigación and FEDER-EU (Project RTI2018101599-B-C21).

\section{Bibliography}

Bell, N. C., Minelli, C., Tompkins, J., Stevens, M. M., \& Shard, A. G. (2012). Emerging techniques for submicrometer particle sizing applied to stöber silica. Langmuir. https://doi.org/10.1021/la301351k

Bradski, G. The OpenCV Library. Dr Dobb's Journal of Software Tools. 2000

Chen, F., Shi, Z., Neoh, K. G., \& Kang, E. T. (2009). Antioxidant and antibacterial activities of eugenol and carvacrol-grafted chitosan nanoparticles. Biotechnology and Bioengineering. https://doi.org/10.1002/bit.22363

Dickinson, E. (2012). Use of nanoparticles and microparticles in the formation and stabilization of food emulsions. Trends in Food Science and Technology. https://doi.org/10.1016/j.tifs.2011.09.006

Emmerich, J., Tang, Q., Wang, Y., Neubauer, P., Junne, S., \& Maaß, S. (2019). Optical inline analysis and monitoring of particle size and shape distributions for multiple applications: Scientific and industrial relevance. Chinese Journal of Chemical Engineering. https://doi.org/10.1016/j.cjche.2018.11.011

García-Ríos, E., Ruiz-Rico, M., Guillamón, J. M., Pérez-Esteve, É., \& Barat, J. M. (2018). Improved antimicrobial activity of immobilised essential oil components against representative spoilage wine microorganisms. Food Control. https://doi.org/10.1016/j.foodcont.2018.07.005

Gill, A. O., \& Holley, R. A. (2006). Disruption of Escherichia coli, Listeria monocytogenes and Lactobacillus sakei cellular membranes by plant oil aromatics. International Journal of Food Microbiology. https://doi.org/10.1016/j.ijfoodmicro.2005.10.009

Ribes, S., Ruiz-Rico, M., Pérez-Esteve, É., Fuentes, A., \& Barat, J. M. (2019). Enhancing the antimicrobial activity of eugenol, carvacrol and vanillin immobilised on silica supports against Escherichia coli or Zygosaccharomyces rouxii in fruit juices by their binary combinations. LWT. https://doi.org/10.1016/j.lwt.2019.108326

Ribes, S., Ruiz-Rico, M., Pérez-Esteve, É., Fuentes, A., Talens, P., Martínez-Máñez, R., \& Barat, J. M. (2017). Eugenol and thymol immobilised on mesoporous silica-based material as an innovative antifungal system: Application in strawberry jam. Food Control. https://doi.org/10.1016/j.foodcont.2017.06.006

Ruiz-Rico, M., Pérez-Esteve, É., Bernardos, A., Sancenón, F., Martínez-Máñez, R., Marcos, M. D., \& Barat, J. M. (2017). Enhanced antimicrobial activity of essential oil components 
immobilized on silica particles. Food Chemistry.

https://doi.org/10.1016/j.foodchem.2017.04.118

Śliwa, T., Jarzębski, M., \& Szutkowski, K. (2015). Nanoparticle Tracking Analysis of Latex Standardized Beads. Current Topics in Biophysics. https://doi.org/10.2478/ctb-2014-0074

Verdú, S., Barat, J. M., \& Grau, R. (2019a). Laser backscattering imaging as a non-destructive quality control technique for solid food matrices: Modelling the fibre enrichment effects on the physico-chemical and sensory properties of biscuits. Food Control. https://doi.org/10.1016/j.foodcont.2019.02.004

Verdú, S., Barat, J. M., \& Grau, R. (2019b). Non destructive monitoring of the yoghurt fermentation phase by an image analysis of laser-diffraction patterns: Characterization of cow's, goat's and sheep's milk. Food Chemistry, 274(July 2018), 46-54. https://doi.org/10.1016/j.foodchem.2018.08.091

Verdú, S., Pérez, A. J., Barat, J. M., \& Grau, R. (2018). Laser backscattering imaging as a control technique for fluid foods: Application to vegetable-based creams processing. Journal of Food Engineering, 241(May 2018), 58-66. https://doi.org/10.1016/j.jfoodeng.2018.08.003

Wang, W., Wen, Y., Lu, J. Q., Zhao, L., Al-Qaysi, S. A., \& Hu, X. H. (2019). Rapid classification of micron-sized particles of sphere, cylinders and ellipsoids by diffraction image parameters combined with scattered light intensity. Journal of Quantitative Spectroscopy and Radiative Transfer. https://doi.org/10.1016/j.jqsrt.2018.12.010

Yin, L. (2012). Dynamic Light Scattering. In Nanotechnology Research Methods for Foods and Bioproducts. https://doi.org/10.1002/9781118229347.ch8 